\title{
Identification of Arthrinium marii as Causal Agent of Olive Tree Dieback in Apulia (Southern Italy)
}

\author{
Donato Gerin, ${ }^{1}$ Franco Nigro, ${ }^{1,2}$ Francesco Faretra, ${ }^{1,2, \dagger}$ and Stefania Pollastro ${ }^{1,2}$ \\ ${ }^{1}$ Department of Soil, Plant and Food Sciences, University of Bari Aldo Moro, Bari 70126, Italy \\ ${ }^{2}$ Selge Network, University of Bari Aldo Moro, Bari 70126, Italy
}

\begin{abstract}
Olive (Olea europaea L. var. sativa) is one of the most economically important tree crops grown in the Mediterranean basin. Arthrinium Kunze ex Fr. (teleomorph: Apiospora Sacc.) is a widespread fungal genus, and Arthrinium marii Larrondo \& Calvo is a ubiquitous species, found in algae, soil, plants, and agricultural communities. A. marii was isolated from olive trees showing dieback from orchards located in Andria and in Fasano, Brindisi (Apulia, southern Italy) and identified based on morphological features and molecular analysis of four genomic regions (ITS, TUB2, TEF1, and

$L S U)$. Two-year-old olive plants artificially inoculated with three representative $A$. marii isolates showed complete dieback within 6 months, and the fungus was reisolated, satisfying Koch's postulates. This is the first report of $A$. marii causing dieback on olive trees that could represent an important threat for olive cultivation.

Keywords: Arbosana, foliar chlorosis, ITS, LSU sequence, TUB2 and TEF1 sequences, withering and twig defoliations
\end{abstract}

Olive (Olea europaea L. var. sativa) is an economically important tree crop of Mediterranean countries, and the European Union is the leading world producer of olive oil and drupes (Food and Agriculture Organization of the United Nations 2017).

Although olive trees are very resistant to environmental stresses, several biotic entities can compromise their health. Fungal species as those belonging to the genus Verticillium are well known as vascular pathogens causing tree wilt, and fungi such as Botryosphaeriaceae spp. (Diplodia spp., Dothiorella iberica, Lasiodiplodia theobromae, Neofusicoccum mediterraneum), Cytospora oleina, Eutypa lata, Phoma incompta, Phaeomoniella chlamydospora, and Phaeoacremonium spp. are associated with olive tree decline, dieback, and cankers and more recently have acquired great importance worldwide (Carlucci et al. 2013, 2015; Ivic et al. 2010; Kaliterna et al. 2012; Moral et al. 2010; Nigro et al. 2013, 2014; Rhouma et al. 2010; Romero et al. 2005; Tosi and Natalini 2009; Úrbez-Torres et al. 2013).

A new olive cropping system consisting of intensive or superintensive plantings is expanding worldwide in order to increase productivity and profits (Tous et al. 2010). The plant density of 140 trees per hectare in traditional growing systems has become up to 400 and 2,500 trees per hectare, respectively, in intensive and superintensive growing systems (Russo et al. 2016).

These systems are characterized by different microclimatic conditions because they need more intensive use of irrigation and fertilization than traditional growing systems and by mechanical pruning and harvesting; consequently, the trees are more susceptible to diseases and pests (Graniti et al. 2011). In fact, in intensive and superintensive growing systems Pseudomonas savastanoi pv. savastanoi increases

${ }^{\dagger}$ Corresponding author: F. Faretra; francesco.faretra@uniba.it

Funding: This research was partially carried out in the framework of the projects "Laboratory network for the selection, characterization and conservation of germplasm and for preventing the spread of economically-relevant and quarantine pests (SELGE) no. 14," funded by the Apulia Region, PO FESR 2007-2013, Axis I, Line of intervention 1.2., Action 1.2.1; and Epidemiology, genetics of plant pathogens and development of molecular diagnostic methods granted by the University of Bari.

The author(s) declare no conflict of interest.

Accepted for publication 19 October 2019.

(C) 2020 The American Phytopathological Society on branches and twigs because of the injuries caused by crop management machinery (Tous et al. 2010). Additionally, an increase in Verticillium wilt has been seen (Jiménez-Díaz et al. 2011; LópezEscudero and Mercado-Blanco 2011).

In 2018 , dieback of about $30 \%$ of trees was observed in three 2year-old olive orchards of cultivar Arbosana located in Andria and in Fasano, Brindisi (Apulia, southern Italy), and this study explored the causes of the dieback.

\section{Materials and Methods}

Sampling and fungal isolation. About $30 \%$ of olive trees (Arbosana) showing dieback were observed in three 2-year-old orchards located in Andria and in Fasano, Brindisi (Apulia, southern Italy). Ten representative symptomatic trees per orchard were randomly sampled and analyzed for wood symptoms in comparison with the healthy ones. Discolored wood tissues were surface-disinfested by submerging them for $2 \mathrm{~min}$ in $2 \%$ sodium hypochlorite solution, washed twice with sterile water, air dried, and then placed onto potato dextrose agar (PDA, infusion from $200 \mathrm{~g}$ of peeled and sliced potatoes kept at $60^{\circ} \mathrm{C}$ for $1 \mathrm{~h}, 20 \mathrm{~g}$ of dextrose, adjusted to $\mathrm{pH} 6.5,20 \mathrm{~g}$ of agar, per liter) plates. Plates were incubated at $24 \pm 1{ }^{\circ} \mathrm{C}$ in the dark, and after 5 days Arthrinium sp. pure cultures were obtained. Three representative monoconidial isolates (DiSSPA_A1, DiSSPA_A2, and DiSSPA_A3) were obtained from pure cultures and used for the subsequent analyses.

Morphology and pathogenicity assay. Morphometric characters were assessed on 10-day-old colonies grown on PDA and malt extract agar (MEA; $20 \mathrm{~g}$ of malt extract and $20 \mathrm{~g}$ of agar, per liter) at $25 \pm 1^{\circ} \mathrm{C}$ according to the synoptic keys proposed for Arthrinium species identification (Crous and Groenewald 2013), and conidia were quantified on the same plates, according to the method described by Crespo-Sempere et al. (2013). For each medium and isolate three replicated plates were used, and data were expressed as number of conidia $\times 10^{4} / \mathrm{mm}^{2}$ of colony. Three hundred globose to elongate ellipsoid conidia were measured for each of the three monoconidial isolates DiSSPA_A1 to A3 with an optical microscope equipped with an ocular micrometer.

The pathogenicity of the DiSSPA_A1 to A3 isolates was investigated by artificial inoculation assays of 2-year-old olive tree cultivars Arbosana and Cellina di Nardò. Two different inoculation methods based on root (method 1) and wood inoculation (method 2) were compared. Briefly, for the method 1, isolates were cultured for 12 days on PDA at $25 \pm 1^{\circ} \mathrm{C}$ in the dark, and conidia were scraped from the colony surface, suspended in sterile water with $0.05 \%$ Tween 20, and filtered on rayon-polyester filtration material to remove mycelium fragments. Roots of 2 -year-old olive trees were 
opportunely cut at $20 \mathrm{~cm}$ in length, fully immersed in 2.5 liters of a suspension containing $5 \times 10^{4} \mathrm{conidia} / \mathrm{ml}$, and maintained overnight at $25 \pm 1^{\circ} \mathrm{C}$ before planting in $10-\times 10-\times 17-\mathrm{cm}$ plastic pots containing a sand-lime-peat soil mixture. For the method 2, mycelial plugs (4 mm diameter) from the edge of 15-day-old colonies on PDA were placed in artificial wounds ( $5 \mathrm{~mm}$ long and $3 \mathrm{~mm}$ deep) under stem bark of olive trees potted as previously described, at $10 \mathrm{~cm}$ height from the crown and then protected with a layer of sealing film. Sterile water (method 1) and sterile PDA medium plugs (method 2) were used as the mock-inoculated controls, and six replicated plants were used for each treatment. Plants were maintained in the greenhouse $\left(25 \pm 2{ }^{\circ} \mathrm{C}\right.$; 16 -h daylight photoperiod) and at 6 months after inoculation (MAI), wood and roots of both the cultivars were inspected for internal symptoms with a destructive assay by dissecting the plants in three portions (about $10 \mathrm{~cm}$ each). The reisolation assay was performed by collecting from each portion five pieces (moving for each about $2 \mathrm{~cm}$ from bottom to top) of woody tissue surface disinfested as reported above and placed on PDA plates. Additionally, at 2, 4, and 6 MAI, the top length (budding) was measured, and canopy symptoms were assessed via an empirical scale of four classes $(0=$ healthy tree, $1=\leq 25 \%$ of the tree showed symptoms [foliar chlorosis and necrosis], $2=26$ to $50 \%$ of the tree showed symptoms, $3=51$ to $75 \%$ of the tree showed symptoms, and $4=76$ to $100 \%$ of the tree showed symptoms). Data were used to calculate the McKinney index (MKI) according to the following formula (McKinney 1923):

$$
\mathrm{MKI}=[\Sigma(f \times v)] /(n \times N) \times 100
$$

where $f$ is the number of symptomatic trees, $v$ is the value of each class, $N$ is the total number of examined trees, and $n$ is the highest value of the classes occurring in the empirical scale.

Data were analyzed by one-way analysis of variance (ANOVA) followed by Tukey's honestly significant difference test in CoStat software at the significance level $P \leq 0.05$. In terms of MKI, no differences were observed for inoculated trees among cultivars and isolates in preliminary two-way ANOVAs, so all data were used as biological replicates in comparing inoculated with mock-inoculated trees.

Molecular and phylogenetic analysis. Genomic DNA of the three monoconidial isolates was extracted according to the cetyl trimethylammonium bromide (CTAB)-based protocol described by De Miccolis Angelini et al. (2010). Briefly, 2-day-old fungal mycelium was collected from sterile cellophane overlapped to PDA plates. Mycelium, powdered under liquid nitrogen, was mixed with $600 \mu \mathrm{l}$ of CTAB buffer (100 mM of Tris-Cl, pH 8.0; $1.4 \mathrm{M}$ of NaCl; $20 \mathrm{mM}$ of EDTA, $\mathrm{pH} 8.0 ; 2 \%$ cetyldimethylethylammonium bromide [wt/ vol]; $0.2 \% \beta$-mercaptoethanol [vol/vol]). Samples were frozen and thawed three times with liquid nitrogen and a water bath at $75^{\circ} \mathrm{C}$, and then incubated at $75^{\circ} \mathrm{C}$ for $1 \mathrm{~h}$. After chloroform extraction, the clear supernatant was transferred to a new tube and precipitated with isopropanol. After $30 \mathrm{~min}$ at $-80^{\circ} \mathrm{C}$ the pellet, collected by centrifugation at 14,000 rpm for $15 \mathrm{~min}$, was washed with cold $70 \%$ ethanol, air dried, dissolved in TE (10 mM of Tris-Cl; $1 \mathrm{mM}$ of EDTA, $\mathrm{pH} 8$ ), treated with $0.1 \mu \mathrm{g} / \mu \mathrm{l}$ of DNAase-free pancreatic RNAase for $2 \mathrm{~h}$ at $37^{\circ} \mathrm{C}$, and finally precipitated by the addition of 0.6 volumes of $5 \mathrm{M}$ of ammonium acetate and 2 volumes of cold absolute ethanol. The final DNA pellet, washed with $70 \%$ ethanol and air dried, was dissolved in ultrapure water and stored at $-80^{\circ} \mathrm{C}$ until use. DNA was quantified with a spectrophotometer and stored at $-20^{\circ} \mathrm{C}$ until use.
The ITS1-5.8S-ITS2 rDNA internal transcribed spacer (ITS) region, translation elongation factor 1- $\alpha$ (TEF1), $\beta$-tubulin (TUB2), and large subunit $(L S U)$ of rDNA were amplified with the primers listed in Table 1. Amplifications were performed in $50 \mu \mathrm{L}$ of reaction volume containing $1 \times$ buffer, $2.5 \mathrm{mM}$ of $\mathrm{MgCl}_{2}, 100 \mathrm{nM}$ of each $\mathrm{dNTP}, 100 \mathrm{nM}$ of each primer, $1 \mathrm{U}$ of Taq polymerase, and $100 \mathrm{ng}$ of DNA template. PCR program consisted of an initial denaturation $\left(94^{\circ} \mathrm{C}, 3 \mathrm{~min}\right), 35$ cycles made up by denaturation $\left(94^{\circ} \mathrm{C}, 1 \mathrm{~min}\right)$, annealing $\left(58^{\circ} \mathrm{C}, 30 \mathrm{~s}\right)$, and extension $\left(72^{\circ} \mathrm{C}, 1 \mathrm{~min}\right)$, followed by a final extension $\left(72^{\circ} \mathrm{C}, 7 \mathrm{~min}\right)$.

PCR products were visualized on a $1.5 \%$ agarose gel $(110 \mathrm{~V}$ for $110 \mathrm{~min}$ ) and custom sequenced. Sequences were singularly analyzed for high sequence similarity through BLASTn by searching in the entire nucleotide collection database of GenBank (https:// www.ncbi.nlm.nih.gov) and also limiting the search to the sequences from type material.

All ITS, TEF1, and TUB2 sequences of Arthrinium sp. (GenBank accession numbers in Table 2) were previously aligned separately in SeqMan Pro software (alignment parameters: match size $=5$, minimum match percentage $=10$, maximum gaps per kilobase in sequence $=130$ ), and only the sequences of 33 isolates (including 9 type specimens) that showed $\geq 80 \%$ identity with those of isolates DiSSPA_A1 to A3 were trimmed with SeqMan Pro Software to obtain the concatenated ITS-TEF1-TUB2 sequences. The $L S U$ sequences were excluded because only a few Arthrinium sp. sequences were available. Phylogenetic analyses were conducted in MEGA version 6 (Tamura et al. 2013) using the maximum parsimony (MP) and maximum likelihood (ML) methods. The MP tree was obtained via the Subtree Pruning and Regrafting algorithm with search level 1, in which the initial trees were obtained by the random addition of sequences (10 replicates). For ML analysis, MEGA was used to infer the best model of nucleotide substitution for the dataset via the Tamura-Nei model and the nearest neighbor interchange heuristic search method. For both MP and ML, the branch stability was determined by 1,000 bootstrap replicates. Nigrospora zimmermanii ITS-TEF1-TUB2 concatenated sequence was used as the outgroup.

\section{Results}

Symptom description, fungal isolation, and pathogenicity. Symptomatic trees exhibited foliar chlorosis and twig defoliations. Wood discolorations were observed on transversal and longitudinal wood sections of branches and twigs, and colonies morphologically referable to Arthrinium sp. were predominantly obtained on PDA (Fig. 1).

Olive trees (Arbosana and Cellina di Nardò) were artificially inoculated with the three Arthrinium marii isolates DiSSPA_A1 to A3. Exclusively on A. marii-inoculated trees, no budding was observed up to $6 \mathrm{MAI}$, when complete dieback occurred. Conversely, noninoculated control trees grew about $10 \mathrm{~cm}$ per month (Fig. 2). At all times, no statistically significant differences between isolates and cultivar were ascertained ( $F$ and $P$ always $\leq 1.6$ and $\geq 0.2$, respectively) by analyses of inoculated trees. On the contrary, inoculated and mock-inoculated trees differed statistically in both root and wood inoculation assays (Table 3 ). The MKI was $0.0 \%$ on noninoculated control plants; $33.3,63.9$, and $86.1 \%$ on root-inoculated trees; and $19.4,38.9$, and $63.9 \%$ on wood-inoculated ones at 2 , 4 , and 6 MAI, respectively.

Table 1. Primers used in this study

\begin{tabular}{llll}
\hline Target gene or region & Primer name & \multicolumn{1}{c}{ Primer sequence $\left(\mathbf{5}^{\prime} \mathbf{- 3}^{\prime}\right)$} & References \\
\hline ITS1-5.8S rDNA-ITS2 region & ITS5 & GGAAGTAAAAGTCGTAACAAGG & White et al. 1989 \\
& ITS4 & TCCTCCGCTTATTGATATGC & Carbone and Kohn 1999 \\
Translation elongation factor 1-a & EF1-728F & CATCGAGAAGTTCGAGAAGG & Glass and Donaldson 1995 \\
& EF1-986R & TACTTGAAGGAACCCTTACC & O'Donnell and Cigelnik 1997 \\
$\beta$-tubulin & T1 & AACATGCGTGAGATTGTAAGT & Vilgalys and Sun 1994 \\
& Bt2b & ACCCTCAGTGTAGTGACCCTTGGC & \\
Large subunit rDNA region & LROR & ACCCGCTGAACTTAAGC & \\
& LR5 & TCCTGAGGGAAACTTCG & \\
\hline
\end{tabular}


Table 2. Details of Arthrinium spp. strains used in the BLASTn and phylogenetic analysis

\begin{tabular}{|c|c|c|c|c|c|c|}
\hline \multirow[b]{2}{*}{ Species } & \multirow[b]{2}{*}{ Strain $^{x}$} & \multirow[b]{2}{*}{ Host } & \multirow[b]{2}{*}{ Country } & \multicolumn{3}{|c|}{ GenBank accession numbers $^{y}$} \\
\hline & & & & ITS & $T U B 2$ & TEF1 \\
\hline Arthrinium arundinis & CBS 464.83 & Phragmites australis & Netherlands & KF144888.1 & KF144979.1 & KF145021.1 \\
\hline A. arundinis & LC7277 & Bambusa sp. & China & KY494750.1 & KY705218.1 & KY705146.1 \\
\hline A. arundinis & EGG3 & Lasioptera donacis & France & MF627422.1 & MF627424.1 & MF627423.1 \\
\hline Arthrinium bambusae & LC7106 & Bambusa sp. & China & KY494719.1 & KY705187.1 & KY705117.1 \\
\hline A. bambusae & LC7113 & Bambusa sp. & China & KY494720.1 & KY705188.1 & KY806205.1 \\
\hline A. bambusae & LC7124 & Bambusa sp. & China & KY494727.1 & KY705195.1 & KY806206.1 \\
\hline Arthrinium camelliae-sinensis & LC8181 & Brassica rapa subsp. oleifera & China & KY494761.1 & KY705229.1 & KY705157.1 \\
\hline A. camelliae-sinensis & LC5007 & Camellia sinensis & China & KY494704.1 & KY705173.1 & KY705103.1 \\
\hline Arthrinium dichotomanthi & LC8175 & Dichotomanthes tristaniicarpa & China & KY494755.1 & KY705223.1 & KY705151.1 \\
\hline A. dichotomanthi & LC8176 & Dichotomanthes tristaniicarpa & China & KY494756.1 & KY705224.1 & KY705152.1 \\
\hline A. dichotomanthi & LC4950 & Dichotomanthes tristaniicarpa & China & KY494697.1 & KY705167.1 & KY705096.1 \\
\hline Arthrinium gaoyouense & CFCC52301 & Phragmites australis & China & MH197124 & МH236789.1 & MH236793.1 \\
\hline A. gaoyouense & CFCC52302 & Phragmites australis & China & MH197125 & MH236790.1 & MH236794.1 \\
\hline Arthrinium guizhouense & LC5322 & Air & China & KY494709.1 & KY705178.1 & KY705108.1 \\
\hline A. guizhouense & LC5318 & Air & China & KY494708.1 & KY705177.1 & KY705107.1 \\
\hline Arthrinium hydei & LC7103 & Bambusa sp. & China & KY494715.1 & KY705183.1 & KY705114.1 \\
\hline A. hydei & CBS 114990 & Bambusa tuldoides & China & KF144890.1 & KF144982.1 & KF145024.1 \\
\hline Arthrinium jiangxiense & LC2831 & Bambusa sp. & China & KY494686.1 & KY806201.1 & KY705085.1 \\
\hline A. jiangxiense & LC4577 & Maesa sp. & China & KY494693.1 & KY705163.1 & KY705092.1 \\
\hline A. jiangxiense & LC5394 & Soil & China & KY494711.1 & KY705180.1 & KY705110.1 \\
\hline Arthrinium kogelbergense & CBS 117206 & Unknown algae & Croatia & KF144895.1 & KF144987.1 & KF145029.1 \\
\hline A. kogelbergense & CBS 113333 & Restionaceae & South Africa & KF144893.1 & KF144985.1 & KF145027.1 \\
\hline A. kogelbergense & CBS 114734 & Uncus gerardii & Sweden & KF144894.1 & KF144986.1 & KF145028.1 \\
\hline Arthrinium malaysianum & CBS 102053 & Macaranga hullettii & Malaysia & KF144896.1 & KF144988.1 & KF145030.1 \\
\hline A. malaysianum & CBS 251.29 & Cinnamomum camphora & Unknown & KF144897.1 & KF144989.1 & KF145031.1 \\
\hline Arthrinium marii & CPC 18904 & Phragmites australis & Italy & KF144902.1 & KF144994.1 & KF145036.1 \\
\hline A. marii & CBS 497.90 & - & Spain & MH862232.1 & KF144993.1 & KF145035.1 \\
\hline A. marii & CBS 200.57 & Beta vulgaris & Netherlands & KF144900.1 & KF144992.1 & KF145034.1 \\
\hline A. marii & CBS 114803 & Pseudosasa hindsii & China & KF144899.1 & KF144991.1 & KF145033.1 \\
\hline A. marii $^{\mathrm{z}}$ & DiSSPA_A1 & Olea europaea & Italy & MK602320.1 & MK614695.1 & MK645472.1 \\
\hline A. marii $^{\mathrm{z}}$ & DiSSPA_A1 & Olea europaea & Italy & MK602321.1 & MK614696.1 & MK645473.1 \\
\hline A. marii $^{\mathrm{z}}$ & DiSSPA_A1 & Olea europaea & Italy & MK602322.1 & MK614697.1 & MK645474.1 \\
\hline Arthrinium obovatum & LC8177 & Lithocarpus sp. & China & KY494757.1 & KY705225.1 & KY705153.1 \\
\hline A. obovatum & LC8178 & Lithocarpus sp. & China & KY494758.1 & KY705226.1 & KY705154.1 \\
\hline A. obovatum & LC4940 & Lithocarpus sp. & China & KY494696.1 & KY705166.1 & KY705095.1 \\
\hline Arthrinium ovatum & CBS 115042 & Arundinaria hindsii & China & KF144903.1 & KF144995.1 & KF145037.1 \\
\hline Arthrinium phaeospermum & CBS 142.55 & Soil & Japan & KF144908.1 & KF145000.1 & KF145042.1 \\
\hline A. phaeospermum & CBS 114318 & Hordeum vulgare & Iran & KF144907.1 & KF144999.1 & KF145041.1 \\
\hline A. phaeospermum & CBS 114317 & Hordeum vulgare & Iran & KF144906.1 & KF144998.1 & KF145040.1 \\
\hline Arthrinium phragmites & CPC: 18900 & Phragmites australis & Italy & KF144909.1 & KF145001.1 & KF145043.1 \\
\hline Arthrinium pseudoparenchymaticum & LC8173 & Bambusa sp. & China & KY494753.1 & KY705221.1 & KY705149.1 \\
\hline A. pseudoparenchymaticum & LC8174 & Bambusa sp. & China & KY494754.1 & KY705222.1 & KY705150.1 \\
\hline A. pseudoparenchymaticum & LC7234 & Bambusa sp. & China & KY494743.1 & KY705211.1 & KY705139.1 \\
\hline Arthrinium pseudospegazzinii & CBS 102052 & Macaranga hullettii & Malaysia & KF144911.1 & KF145002.1 & KF145045.1 \\
\hline Arthrinium pterospermum & CPC:20193 & Lepidosperma gladiatum & Australia & KF144913.1 & KF145004.1 & KF145046.1 \\
\hline Arthrinium rasikravindrae & LC5449 & Unknown & China & KY494713.1 & KY705182.1 & KY705112.1 \\
\hline A. rasikravindrae & LC7115 & Bambusa sp. & China & KY494721.1 & KY705189.1 & KY705118.1 \\
\hline A. rasikravindrae & LC7117 & Bambusa sp. & China & KY494722.1 & KY705190.1 & KY705119.1 \\
\hline Arthrinium sacchari & CBS 372.67 & Air & Unknown & KF144918.1 & KF145007.1 & KF145049.1 \\
\hline A. sacchari & CBS 664.74 & Soil & Netherlands & KF144919.1 & KF145008.1 & KF145050.1 \\
\hline A. sacchari & CBS 301.49 & Bambusa sp. & Indonesia & KF144917.1 & KF145006.1 & KF145048.1 \\
\hline Arthrinium saccharicola & CBS 831.71 & Unknown & Netherlands & KF144922.1 & KF145012.1 & KF145054.1 \\
\hline A. saccharicola & CBS 463.83 & Phragmites australis & Netherlands & KF144921.1 & KF145011.1 & KF145053.1 \\
\hline A. saccharicola & CBS 191.73 & Unknown & Netherlands & KF144920.1 & KF145009.1 & KF145051.1 \\
\hline Arthrinium subroseum & LC7215 & Bambusa sp. & China & KY494740.1 & KY705208.1 & KY705136.1 \\
\hline A. subroseum & LC7291 & Bambusa sp. & China & KY494751.1 & KY705219.1 & KY705147.1 \\
\hline Arthrinium thailandicum & LC5630 & Unknown & China & KY494714.1 & KY806200.1 & KY705113.1 \\
\hline Arthrinium xenocordella & CBS 478.86 & Soil & Zimbabwe & KF145013.1 & KF144925.1 & KF145055.1 \\
\hline A. xenocordella & LC3486 & Camellia sinensis & China & KY494687.1 & KY705158.1 & KY705086.1 \\
\hline
\end{tabular}


At the end of the experiment (6 MAI), arthrinium-like colonies were always reisolated from discolored woody tissues, 25 to $30 \mathrm{~cm}$ away from the inoculation point (stem or roots) of the inoculated plants (Arbosana and Cellina di Nardò). Moreover, about 30\% of roots showed discolorations only in the root-inoculated trees with A. marii. No arthrinium-like colonies were obtained from noninoculated control trees. Other fungi morphologically characterized, such

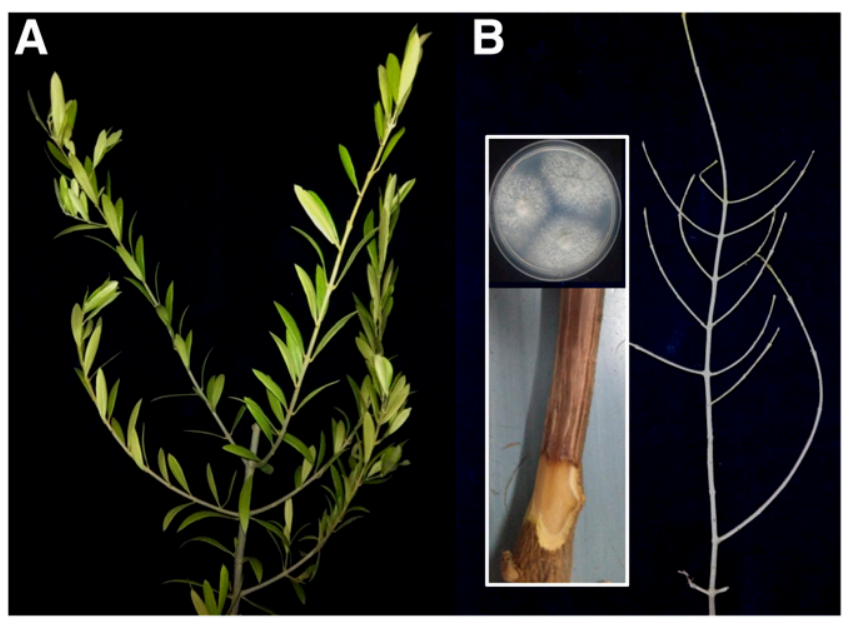

Fig. 1. Apical shoots of A, healthy and B, symptomatic 2-year-old olive plants along with wood discoloration from which the 7-day-old Arthrinium marii colonies were obtained on potato dextrose agar (PDA).

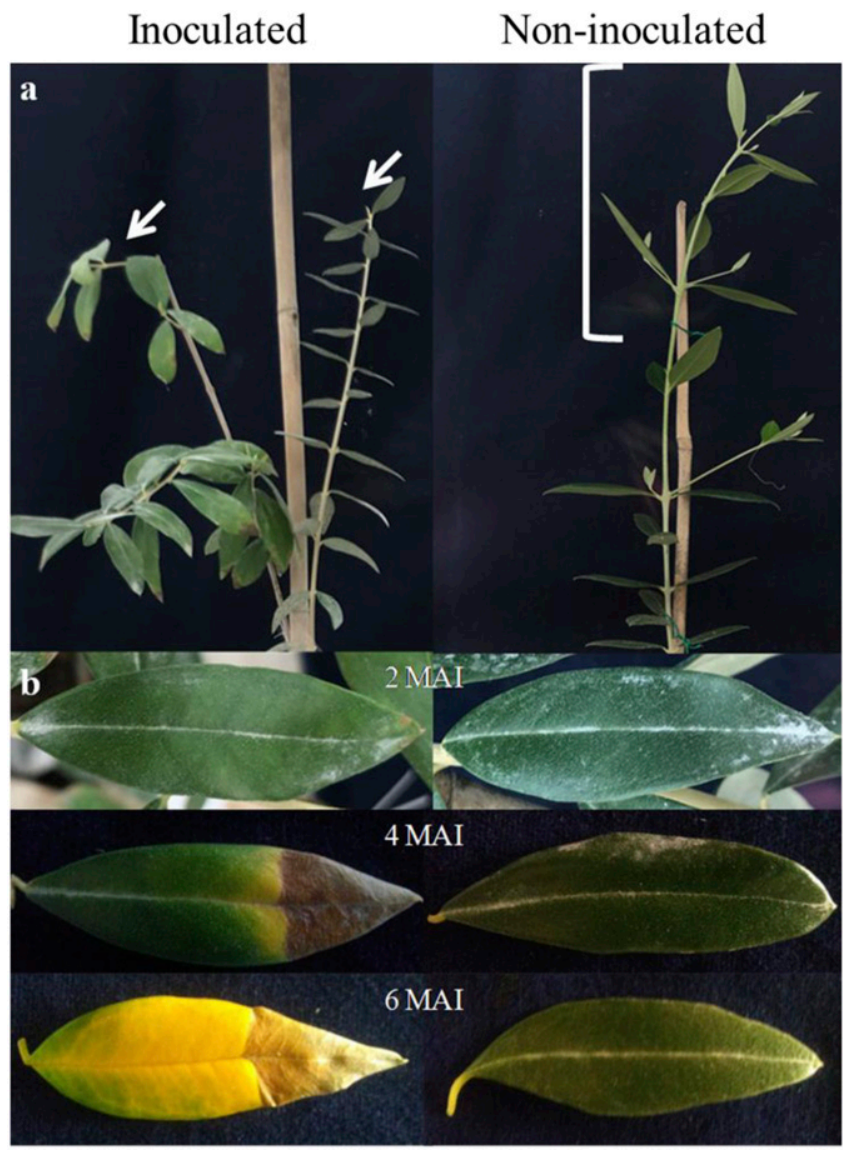

Fig. 2. Olive plants inoculated or not with Arthrinium marii. a, Detail of budding in $A$. marii inoculated and noninoculated plants at 2 months after inoculation (MAI). b, General status of leaves at 2, 4, and 6 MAI of $A$. marii inoculated and noninoculated trees. Arrows indicate the absence of budding in the inoculated tree, and bracket marks indicate budding of noninoculated tree. as Trichoderma, Alternaria, and Cladosporium, were isolated from all the plants, irrespective of artificial inoculation method.

Morphology of $A$. marii isolates. The morphology of $A$. marii isolates was observed on PDA and MEA after 10 days of incubation at $25^{\circ} \mathrm{C}$ in the dark. On both media, colonies were flat, spreading with sparse aerial mycelium. On PDA, cultures of the three isolates showed an olivaceous gray surface and olivaceous gray patches on the reverse surface. On MEA, the cultures appeared with a whitegray surface and gray patches on the reverse surface (Fig. 3A).

Mycelium consisted of smooth, hyaline, branched, and septate hyphae measuring 2 to $4 \mu \mathrm{m}$ in diameter. Fifteen-day-old PDA cultures exhibited conidiophores in form of conidiogenous cells that were aggregated in clusters on brown (Fig. 3B), smooth, and ampulliform hyphae, producing conidia (Fig. 3C, D). Conidia were brown, smooth, granular, globose to elongate ellipsoid, and measuring 6 to $13 \mu \mathrm{m}$ in diameter. Brown, elongated, sterile cells (average $22 \times 5 \mu \mathrm{m}$ ), typical of some Arthrinium species (Samuels et al. 1981), were also observed (Fig. 3E).

Finally, for all isolates, the conidia production was higher on MEA (3.2 to $5.0 \times 10^{4}$ conidia per square millimeter) with respect to PDA (5.5 to $8.4 \times 10^{4}$ conidia per square millimeter) (Table 4).

Molecular and phylogenetic analysis. Sequences of 567, 833, 262, and 727 were obtained by sequencing the partial ITS, $L S U$, $T E F 1$, and TUB2 regions, respectively, of the three $A$. marii isolates DiSSPA_A1 to A3 (Table 2).

BLASTn analysis against the entire nucleotide collection database of GenBank revealed that all sequences showed 99 to $100 \%$ identity (coverage $=99$ to $100 \%$, e-value $=0.0$ ) with different Arthrinium species. In more detail, for the three isolates the ITS region showed $100 \%$ similarity with A. marii, Arthrinium sp., and Arthrinium phaeospermum (e.g., KF144900.1, MH355544.1, and KY081461.1), the LSU showed $99 \%$ similarity with A. marii, Arthrinium sp., and Arthrinium arundinis (e.g., KF144946.1, MH109530.1, and KF993394.1), the TEF1 showed 99\% similarity with A. marii (e.g., KF145034.1), and the TUB2 showed $100 \%$ similarity with A. marii and Arthrinium hispanicum (KF144992.1 and AB220289.1). Although none of them was a type specimen, $A$. marii was the only species showing the maximum identity for all four analyzed sequences.

Additionally, with the BLASTn analysis only against sequences from type material, all sequences (ITS, $L S U, T E F 1$, and TUB2) of the three isolates showed 99 to $100 \%$ identity (coverage $=99$ to $100 \%$, e-value $=0.0$ ) with $A$. marii type specimen (CBS 497.90, accession numbers in Table 2). The ITS and $L S U$ regions of the three isolates showed identities $>99 \%$, although less than A. marii CBS 497.90, also with Arthrinium guizhouense, Arthrinium longistromum, and Arthrinium pseudospegazzinii type specimens (LC5322, MFLU 15-1184, and CBS 102052 strains, respectively). Finally, identities $<96 \%$ with different Arthrinium type specimens were observed for TEF1 and TUB2 regions.

After the first alignment of ITS, TUB2, and TEF1 sequences, 33 of 56 Arthrinium spp. showed identity $>80 \%$ with the DiSSPA_A1 to A3 isolates. The most parsimonious tree obtained by MP analysis of concatenated ITS-TEF1-TUB2 sequences (tree length $=545$, consistency index $=0.608$, retention index $=0.812$, composite index $=$ 0.621 ) is shown in Figure 4. The analysis using the ML and

Table 3. McKinney index for olive trees artificially inoculated with Arthrinium marii ${ }^{\mathrm{z}}$

\begin{tabular}{lrrr}
\hline Inoculation & 2 MAI & 4 MAI & 6 MAI \\
\hline Wood & & & \\
A. marii & $19.4 \pm 2.8 \mathrm{a}$ & $38.9 \pm 4.8 \mathrm{a}$ & $63.9 \pm 2.8 \mathrm{a}$ \\
Control & $0.0 \pm 0.0 \mathrm{~b}$ & $0.0 \pm 0.0 \mathrm{~b}$ & $0.0 \pm 0.0 \mathrm{~b}$ \\
Root & & & \\
A. marii & $33.3 \pm 0.0 \mathrm{a}$ & $63.9 \pm 2.8 \mathrm{a}$ & $86.1 \pm 2.8 \mathrm{a}$ \\
Control & $0.0 \pm 0.0 \mathrm{~b}$ & $0.0 \pm 0.0 \mathrm{~b}$ & $0.0 \pm 0.0 \mathrm{~b}$ \\
\hline
\end{tabular}

${ }^{\mathrm{z}}$ Data represent the mean values \pm standard error. MAI, months after inoculation. For each MAI and inoculation assay, data followed by different letters were statistically significant at probability level $P \leq 0.05$ according to the Tukey test. 
Tamura-Nei model resulted in a tree similar to that obtained with MP analysis, and with the highest log likelihood of -7344.64. Both analyses showed that the concatenated ITS-TEF1-TUB2 sequences of all Arthrinium isolates obtained with this study clustered with $A$. marii type specimen, albeit with support $\leq 91 \%$ (Fig. 4).

\section{Discussion}

In this study we identified $A$. marii as the causal agent of a severe dieback of olive trees observed in 2018 in three 2-year-old olive orchards, Arbosana located in Apulia (southern Italy). The fungus is ubiquitous in nature, and it has been reported in soils, plants, and agricultural communities (Agut and Calvo 2004; Crous and Groenewald 2013; Dai et al. 2016; Gnavi et al. 2017; Oliveira et al. 2012; Senanayake et al. 2015; Sharma et al. 2014; Wang et al. 2018) as well as in green algae (Gnavi et al. 2017). Arthrinium Kunze ex Fr. is an anamorph genus, which has been traditionally linked to the teleomorph genus Apiospora Sacc.

Arthrinium currently comprises 80 species (http://www.index fungorum.org), and several of them have been documented as plant pathogens and endophytes (Mavragani et al. 2007; Sharma et al. 2014; Wang et al. 2018), although information on fungal biology and disease epidemiology is generally scanty. Reports on Arthrinium diseases are quite recent, but different species are known to be pathogens in different host plants, probably because of changes in environmental conditions, cultural practices, or the introduction of new cultivars (Crous and Groenewald 2013; Lo Piccolo et al. 2014; Mavragani et al. 2007). For example, A. arundinis causes brown culm streak of Phyllostachys praecox, is additionally found on different plant hosts in China (e.g., Bambusa sp., Bothrocaryum controversum, Dichotomanthus tristaniaecarpa, Phyllostachys sp., and Osmanthus sp.) (Chen et al. 2014; Wang et al. 2018), and has been isolated from the leaf of Hordeum vulgare, living leaves of Fagus sylvatica in Iran and Switzerland, and rosemary in Iran (Bagherabadi et al. 2014; Crous and Groenewald 2013). Arthrinium sacchari causes damping-off of durum wheat in the semiarid Saskatchewan fields (Mavragani et al. 2007). Arthrinium saccharicola has been isolated from living and dead culms of Phragmites australis and even from the air in the Netherlands and France and from seawater in mangrove habitats in Hong Kong (Crous and Groenewald 2013; Miao et al. 2006). Arthrinium xenocordella is reported as causing fruit blight on Pistacia vera in Italy (Aiello et al. 2018). A. phaeospermum, responsible for culm rot on Phyllostachys viridis ( $\mathrm{Li}$ et al. 2016), was recently demonstrated to be also associated with necrosis of olive leaves, and according to Koch's postulates the pathogenicity was proved only on leaves and not on twigs (Lo Piccolo et al. 2014). In this study, A. marii isolates were collected from branches and twigs of olive trees showing dieback, and they reproduced the disease when artificially inoculated on 2-year-old olive plants, satisfying Koch's postulates. The fungus was shown to be highly virulent; in fact, at $6 \mathrm{MAI}$ it was reisolated 25 to $30 \mathrm{~cm}$ away from the inoculation point. A similar behavior was reported for Botryosphaeria dothidea and Neofusicoccum mediterraneum, causing $\leq 16-\mathrm{cm}$ lesions on olive branches within 1 MAI (Moral et al. 2010, 2017) and for Neofabraea kienholzii and Phlyctema vagabunda, both isolated from shoot

Table 4. Conidia production by Arthrinium marii isolates ${ }^{\mathrm{z}}$

\begin{tabular}{lcc}
\hline & \multicolumn{2}{c}{ Conidia $\left(\mathbf{N o} \times \mathbf{\times} \mathbf{1 0}^{\mathbf{4}} \mathbf{\mathbf { m m } ^ { \mathbf { 2 } } )}\right.$} \\
\cline { 2 - 3 } Isolate & PDA & MEA \\
\hline DiSSPA_A1 & $3.2 \pm 0.4$ & $6.6 \pm 0.9$ \\
DiSSPA_A2 & $5.0 \pm 0.7$ & $8.4 \pm 0.6$ \\
DiSSPA_A3 & $3.2 \pm 0.5$ & $5.5 \pm 0.7$ \\
\hline
\end{tabular}

z PDA, potato dextrose agar; MEA, malt extract agar. Data represent the mean values \pm standard error.

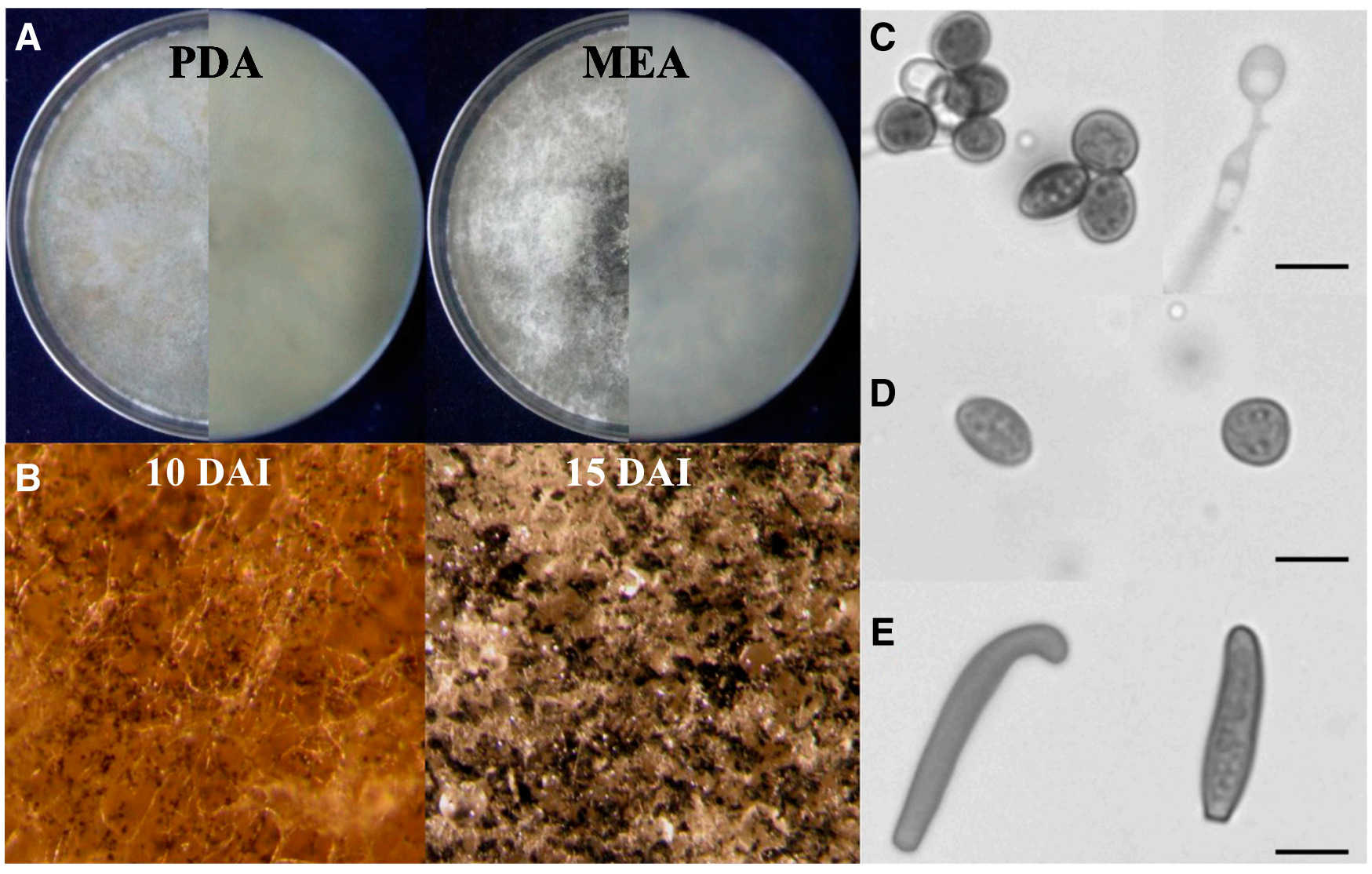

Fig. 3. Morphology of the Arthrinium marii DiSSPA_A1 isolated from olive trees. A, Top and bottom of potato dextrose agar (PDA) and malt extract agar (MEA) cultures. B, details of PDA colony after 10 and 15 days after inoculation (DAl). C, Conidia and conidiogenous cell. D, Conidia. E, Sterile cells. In C, D, and E, bars represent $10 \mu \mathrm{m}$, and observations refer to 15-day-old PDA cultures. 
lesions on olive in California and producing lesions up to $4 \mathrm{~cm}$ in length at $3 \mathrm{MAI}$, when inoculated into 2-year-old detached shoots (Trouillas et al. 2019).

The morphological features of the three isolates DiSSPA_A1 to A3 agreed with those described for A. marii by Crous and Groenewald (2013). In addition to the conidia, produced in higher amounts on MEA than on PDA, all isolates (DiSSPA_A1 to A3) also produced sterile cells; these features are well known for some Arthrinium species, including A. marii (Crous and Groenewald 2013; Samuels et al. 1981).

Examination of morphological features and molecular analysis were both necessary for the appropriate identification of $A$. marii. Recently, a reassessment of Arthrinium sp. was proposed based on the phylogeny of $L S U$ and ITS regions as well as concatenated TUB2-TEF1 sequences (Crous and Groenewald 2013). According to the nucleotide

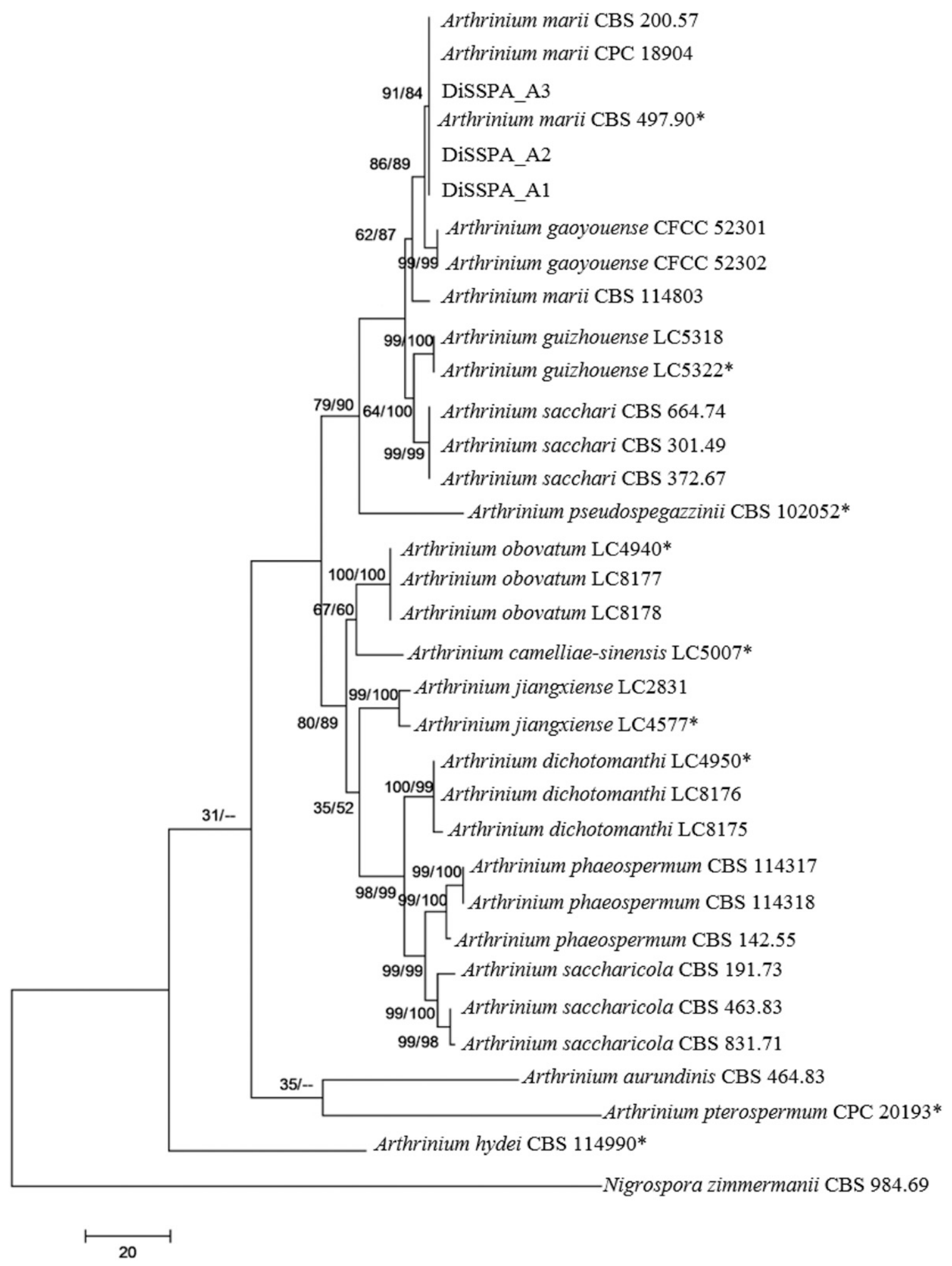

Fig. 4. Phylogenetic tree of concatenated ITS-TEF1-TUB2 sequences of Arthrinium spp. generated by both maximum parsimony (MP) and maximum likelihood (ML) analyses. In the figure the percentage of replicate trees in which the associated taxa clustered together in the bootstrap test (1,000 replicates) are shown on the nodes (MP/ML). Nigrospora zimmermanii was used as the outgroup. ${ }^{*}$ Type specimens. 
BLASTn analysis of ITS, TUB2, TEF1, and $L S U$ gene sequences, A. marii (type specimen and other isolates) was the only species matching with a high identity (99 to 100\%) for all four analyzed sequences. This result was also confirmed by phylogenetic analysis using the concatenated ITS-TEF1-TUB2 sequences. In fact, all isolates (DiSSPA_A1 to A3) clustered with the type specimen of $A$. marii (CBS 497.90), although with a support of $\leq 91 \%$ of the bootstrap test, and they represented a distinct clade in respect to the other species, including the recently described Arthrinium gaoyouense (Jiang et al. 2018). Additionally, our results confirm that strain CBS 114803 , identified as A. marii, seems to represent an independent lineage, as recently reported by Pintos et al. (2019).

In conclusion, to our knowledge this is the first report of $A$. marii, a fungal species that seems ubiquitous in nature, as an agent of severe dieback of olive trees in southern Italy.

The spread of the fungus could represent a serious threat for olive cultivation, because of the challenges of the new planting systems (intensive and superintensive) and because of the introduction into agricultural practice of new varieties more adapted to the new growing system. Generally, the number of fungal species reported as causal agents of trees' decline and dieback is increasing, and the interactions established by different fungal species inhabiting olive trees are unknown (Graniti et al. 2011; Nigro et al. 2013, 2014). Additionally, fertilizers and biostimulants obtained from biomasses of different origin, including algae, are available on the market and commonly applied in agriculture because they are considered eco-friendly, but information on the effect of the algae mycobiota on cultivated plants are scanty. For instance, A. marii is a component of the mycobiota of the Mediterranean green alga Flabellia petiolata (Gnavi et al. 2017) and the calcareous brown alga Padina pavonica (Garzoli et al. 2018). It is crucial to improve basic knowledge on the microbial community interacting in the complex olive pathosystem in order to implement adequate protection strategies.

\section{Literature Cited}

Agut, M., and Calvo, M. A. 2004. In vitro conidial germination in Arthrinium aureum and Arthrinium phaeospermum. Mycopathologia 157:363-367.

Aiello, D., Gulisano, S., Gusella, G., Polizzi, G., and Guarnaccia, V. 2018. First report of fruit blight caused by Arthrinium xenocordella on Pistacia vera in Italy. Plant Dis. 102:1853.

Bagherabadi, S., Zafari, D., and Anvar, F. G. 2014. First report of leaf spot caused by Arthrinium arundinis on rosemary in Iran. J. Plant Pathol. 96:126. http:// www.sipav.org/main/jpp/index.php/jpp/article/view/3218

Carbone, I., and Kohn, L. M. 1999. A method for designing primer sets for speciation studies in filamentous Ascomycetes. Mycologia 91:553-556.

Carlucci, A., Lops, F., Cibelli, F., and Raimondo, M. L. 2015. Phaeoacremonium species associated with olive wilt and decline in southern Italy. Eur. J. Plant Pathol. 141:717-729.

Carlucci, A., Raimondo, M. L., Cibelli, F., Phillips, A. J., and Lops, F. 2013. Pleurostomophora richardsiae, Neofusicoccum parvum and Phaeoacremonium aleophilum associated with a decline of olives in southern Italy. Phytopathol. Mediterr. 52:517-527.

Chen, K., Wu, X. Q., Huang, M. X., and Han, Y. Y. 2014. First report of brown culm streak of Phyllostachys praecox caused by Arthrinium arundinis in Nanjing, China. Plant Dis. 98:1274

Crespo-Sempere, A., Marin, S., Sanchis, V., and Ramos, A. J. 2013. VeA and LaeA transcriptional factors regulate ochratoxin A biosynthesis in Aspergillus carbonarius. Int. J. Food Microbiol. 166:479-486.

Crous, P. W., and Groenewald, J. Z. 2013. A phylogenetic re-evaluation of Arthrinium. IMA Fungus 4:133-154.

Dai, D. Q., Jiang, H. B., Tang, L. Z., and Bhat, D. J. 2016. Two new species of Arthrinium (Apiosporaceae, Xylariales) associated with bamboo from Yunnan, China. Mycosphere 7:1332-1345.

De Miccolis Angelini, R. M., Habib, W., Rotolo, C., Pollastro, S., and Faretra, F. 2010. Selection, characterization and genetic analysis of laboratory mutants of Botryotinia fuckeliana (Botrytis cinerea) resistant to the fungicide boscalid. Eur. J. Plant Pathol. 128:185-199.

Food and Agriculture Organization of the United Nations. 2017. FAOSTAT: Agriculture data collection (crops). http://www.fao.org/faostat/en/\#home

Garzoli, L., Poli, A., Prigione, V., Gnavi, G., and Varese, G. C. 2018. Peacock tail with a fungal cocktail: first assessment of the mycobiota associated with the brown alga Padina pavonica. Fungal Ecol. 35:87-97.

Glass, N. L., and Donaldson, G. C. 1995. Development of primer sets designed for use with the PCR to amplify conserved genes from filamentous ascomycetes. Appl. Environ. Microbiol. 61:1323-1330.
Gnavi, G., Garzoli, L., Poli, A., Prigione, V., Burgaud, G.-A., and Varese, G. C. 2017. The culturable mycobiota of Flabellia petiolata: First survey of marine fungi associated to a Mediterranean green alga. PLoS One 12:e0175941.

Graniti, A., Faedda, R., Cacciola, S. O., and di San Lio, G. M. 2011. Olive diseases in a changing ecosystem. Pages 403-433 in: Olive Diseases and Disorders. L. Schena, G. E. Agosteo, and S. O. Cacciola, eds. Transworld Research Network, Kerala, India.

Ivic, D., Ivanovic, A., Milicevic, T., and Cvjetkovic, B. 2010. Shoot necrosis of olive caused by Phoma incompta. Phytopathol. Mediterr. 49:414-416.

Jiang, N., Li, J., and Tian, C. M. 2018. Arthrinium species associated with bamboo and reed plants in China. Fungal Syst. Evol. 2:1-9.

Jiménez-Díaz, R. M., Olivares-García, C., Landa, B. B., del Mar Jiménez-Gasco, M., and Navas-Cortés, J. A. 2011. Region-wide analysis of genetic diversity in Verticillium dahliae populations infecting olive in southern Spain and agricultural factors influencing the distribution and prevalence of vegetative compatibility groups and pathotypes. Phytopathology 101:304-315.

Kaliterna, J., Milicevic, T., Ivic, D., Bencic, D., and Mesic, A. 2012. First report of Diplodia seriata as causal agent of olive dieback in Croatia. Plant Dis. 96:290.

Li, B. J., Liu, P. Q., Jiang, Y., Weng, Q. Y., and Chen, Q. H. 2016. First report of culm rot caused by Arthrinium phaeospermum on Phyllostachys viridis in China. Plant Dis. 100:1013.

Lo Piccolo, S., Mondello, V., Giambra, S., Conigliaro, G., Torta, L., and Burruano, S. 2014. Arthrinium phaeospermum, Phoma cladoniicola and Ulocladium consortiale, new olive pathogens in Italy. J. Phytopathol. 162:258-263.

López-Escudero, F. J., and Mercado-Blanco, J. 2011. Verticillium wilt of olive: a case study to implement an integrated strategy to control a soil-borne pathogen. Plant Soil 344:1-50.

Mavragani, D. C., Abdellatif, L., McConkey, B., Hamel, C., and Vujanovic, V. 2007. First report of damping-off of durum wheat caused by Arthrinium sacchari in the semi-arid Saskatchewan fields. Plant Dis. 91:469.

McKinney, H. H. 1923. Influence of soil temperature and moisture on infection of wheat seedlings by Helmintosporium sativum. J. Agric. Res. 26:195-218.

Miao, L., Kwong, T. F. N., and Qian, P.-Y. 2006. Effect of culture conditions on mycelial growth, antibacterial activity, and metabolite profiles of the marinederived fungus Arthrinium c.F. saccharicola. Appl. Microbiol. Biotechnol. 72: 1063-1073.

Moral, J., Agustí-Brisach, C., Pérez-Rodríguez, M., Xaviér, C., Raya, M. C., Rhouma, A., and Trapero, A. 2017. Identification of fungal species associated with branch dieback of olive and resistance of table cultivars to Neofusicoccum mediterraneum and Botryosphaeria dothidea. Plant Dis. 101:306-316.

Moral, J., Muñoz-Díez, C., González, N., Trapero, A., and Michailides, T. J. 2010. Characterization and pathogenicity of Botryosphaeriaceae species collected from olive and other hosts in Spain and California. Phytopathology 100: $1340-1351$

Nigro, F., Antelmi, I., and Ippolito, A. 2014. Identification and characterization of fungal species associated with the quick decline of olive. International Symposium on the European Outbreak of Xylella fastidiosa in Olive. J. Plant Pathol. 96:101-102.

Nigro, F., Boscia, D., Antelmi, I., and Ippolito, A. 2013. Fungal species associated with a severe decline of olive in southern Italy. J. Plant Pathol. 95:668.

O’Donnell, K., and Cigelnik, E. 1997. Two divergent intragenomic rDNA ITS2 types within a monophyletic lineage of the fungus Fusarium are non-orthologous. Mol. Phylogenet. Evol. 7:103-116.

Oliveira, I., Pereira, J. A., Lino-Neto, T., Bento, A., and Baptista, P. 2012. Fungal diversity associated to the olive moth, Prays oleae Bernard: a survey for potential entomopathogenic fungi. Microb. Ecol. 63:964-974.

Pintos, Á., Alvarado, P., Planas, J., and Jarling, R. 2019. Six new species of Arthrinium from Europe and notes about A. caricicola and other species found in Carex spp. hosts. MycoKeys 49:15-48.

Rhouma, A., Triki, M. A., Krid, S., Tuset, J. J., and Msallem, M. 2010. First report of a branch dieback of olive trees in Tunisia caused by a Phoma sp. Plant Dis. 94:636.

Romero, M. A., Sánchez, M. E., and Trapero, A. 2005. First report of Botryosphaeria ribis as a branch dieback pathogen of olive trees in Spain. Plant Dis. 89:208.

Russo, C., Cappelletti, G., Nicoletti, G., Di Noia, A., and Michalopoulos, G. 2016 Comparison of European olive production systems. Sustainability 8:825.

Samuels, G. J., McKenzie, E. H. C., and Buchanan, D. E. 1981. Ascomycetes of New Zealand 3. Two new species of Apiospora and their Arthrinium anamorphs on bamboo. N. Z. J. Bot. 19:137-149.

Senanayake, I. C., Maharachchikumbura, S. S., Hyde, K. D., Bhat, J. D., Jones, E. G., McKenzie, E. H., Dai, D. Q., Daranagama, D. A., Dayarathne, M. C., Goonasekara, I. D., Konta, S., Li, W. J., Qiu, J. S., Stadler, M. Wijayawardene, N. N., Xiao, Y. P., Norphanphoun, C., Li, Q., Liu, X. Z., Bahkali, A. H., Kang, J. C., Wang, Y., Wen, T. C., Wendt, L., Xu, J. C., and Camporesi, E. 2015. Towards unraveling relationships in Xylariomycetidae (Sordariomycetes). Fungal Divers. 73:73-144.

Sharma, R., Kulkarni, G., Sonawane, M. S., and Shouche, Y. S. 2014. A new endophytic species of Arthrinium (Apiosporaceae) from Jatropha podagrica. Mycoscience 55:118-123.

Tamura, K., Stecher, G., Peterson, D., Filipski, A., and Kumar, S. 2013. MEGA6: molecular evolutionary genetics analysis version 6.0. Mol. Biol. Evol. 30 $2725-2729$. 
Tosi, L., and Natalini, G. 2009. First report of Eutypa lata causing dieback of olive trees in Italy. Plant Pathol. 58:398.

Tous, J., Romero, A., and Hermoso, J. F. 2010. New trends in olive orchard design for continuous mechanical harvesting. Adv. Hortic. Sci. 24:43-52.

Trouillas, F. P., Nouri, M. T., Lawrence, D. P., Moral, J., Travadon, R., Aegerter, B., and Lightle, D. 2019. Identification and characterization of Neofabraea kienholzii and Phlyctema vagabunda causing leaf and shoot lesions of olive in California. Plant Dis. 103:3018-3030.

Úrbez-Torres, J. R., Peduto, F., Vossen, P. M., Krueger, W. H., and Gubler, W. D. 2013. Olive twig and branch dieback: etiology, incidence, and distribution in California. Plant Dis. 97:231-244.
Vilgalys, R., and Sun, B. L. 1994. Ancient and recent patterns of geographic speciation in the oyster mushroom Pleurotus revealed by phylogenetic analysis of ribosomal DNA sequences. Proc. Natl. Acad. Sci. USA 91: 4599-4603.

Wang, M., Tan, X.-M., Liu, F., and Cai, L. 2018. Eight new Arthrinium species from China. MycoKeys 34:1-24.

White, T. J., Bruns, T., Lee, S. J. W. T., and Taylor, J. L. 1989. Amplification and direct sequencing of fungal ribosomal RNA genes for phylogenetics. Pages 315-322 in: PCR Protocols: A Guide to Methods and Applications. M. A. Innis, D. H. Gelfand, J. J. Sninsky, and T. J. White, eds. Elsevier, Amsterdam, The Netherlands. 